\title{
Abstracts presented at the Laryngology and Rhinology Section Meetings, Royal Society of Medicine, 2 March 2012 and 1 February 2013, London, UK
}

Abstracts for 2012

Are we performing more laryngeal biopsies, and if so, why? A decade of results

S Lim

From Glasgow Royal Infirmary

\section{Introduction}

Laryngeal biopsies are frequently performed in the management of benign, pre-malignant and malignant conditions. Over the past decade, we have observed an escalating trend in the number of biopsies performed, despite the incidence of laryngeal cancer remaining constant during this period.

Aims

To quantify the rate of laryngeal biopsies, and to record the indications for biopsies performed between 2001 and 2010 .

\section{Method}

A retrospective analysis of laryngeal biopsies performed in North Glasgow between 2001 and 2010 was carried out using the North Glasgow Pathology Database.

Results

From 2001 to 2010, 3902 cases involving a laryngeal biopsy were identified. Of those, 888 ( 23 per cent) were diagnosed with squamous cell carcinoma (SCC), 705 (18 per cent) were diagnosed with dysplasia, 831 (21 per cent) had no tumour and the remaining 1478 (38 per cent) were diagnosed with benign pathology.

Between 2001 and 2003, approximately 300 biopsies were performed annually. From 2004 to 2010 , over 400 biopsies were performed annually. This increase was significant (chi-square $=115.0, p<0.001$ ).

Annually, approximately 120 biopsies indicate benign results and 90 are diagnostic indicators of SCC; this rate has remained unchanged over the past decade.

From 2004, there has been a significant increase in biopsies conducted for dysplasia and surveillance of SCC. The rate for the latter has increased 2-3 fold, despite the incidence of SCC remaining constant. This increase was significant $($ chi-square $=71.78, p<0.001)$

\section{Conclusion}

Since 2004, there has been an increase in the number of biopsies performed; however, the incidence rates of SCC and benign disease have remained relatively constant. It is hypothesised that organ preservation strategies, endoscopic resection in early laryngeal cancer, and chemoradiotherapy in advanced head and neck cancer are responsible for this increase in the number of biopsies.

Improved quality of life in adults undergoing tonsillectomy for recurrent tonsillitis

H Powell

From the West Middlesex University Hospital and Southend University Hospital

\section{Objective}

According to a recent survey, two-thirds of primary care organisations now restrict referrals for treatments they deem to be non-urgent or of low clinical value. There is a paucity of studies on patient-reported outcomes following adult tonsillectomy. Our aim was to add to the body of health-related quality of life (QoL) evidence on adult tonsillectomy for recurrent tonsillitis at a time when this intervention is being branded a low priority treatment.

\section{Participants and methods}

We carried out a prospective questionnaire study in two district general hospitals. Forty-one patients were recruited and each completed the pre-operative Short Form 36 questionnaire. All patients were contacted at least one year after tonsillectomy and were asked to complete the same Short Form 36 questionnaire and three additional health-related QoL questions.

\section{Main outcome measures}

These were the pre-operative and one-year post-operative Short Form 36 scores, and the results of the health-related QoL questions.

Results

The mean age of participants was 24 years and 6 months. Assuming a non-normal distribution, a two-tailed $t$-test was used to compare pre-operative and post-operative scores. Both the mean Short Form 36 scores and responses to the health-related QoL questions revealed a significant improvement in QoL. The Short Form 36 summary measures and the total Short Form 36 scores improved significantly $(p<0.01)$.

\section{Conclusions}

The study emphasised the importance of tonsillectomy as a National Health Service treatment for adults with recurrent tonsillitis. The improvement in QoL is likely to confer secondary economic benefits associated with 
fewer general practitioner appointments and missed work days.

\section{Abstracts for 2013}

Airway remodelling in chronic rhinosinusitis: is epithelial-fibroblast signalling to blame?

\section{S Ball, A De Soyza, A Fisher, D Mann, J Wilson}

From Newcastle University and Freeman Hospital, Newcastle upon Tyne

\section{Background}

Chronic rhinosinusitis is one of the most common otolaryngological conditions, yet its pathogenesis remains unclear. Sinonasal epithelial inflammation and airway remodelling are thought to be key steps in the disease development process; however, the mechanisms by which these occur need clarifying.

\section{Aim}

To examine the pro-inflammatory signalling of sinonasal epithelium and fibroblasts as a driver of chronic rhinosinusitis.

\section{Methods}

Carefully phenotyped patients with chronic rhinosinusitis refractory to maximal medical therapy were prospectively recruited along with healthy controls. Ethical approval and informed consent were obtained.

Matched primary nasal epithelial cell brushings and tissue biopsies were harvested from each participant. Primary nasal epithelial cells were grown in submerged culture. The model was extensively characterised and validated with reverse transcription polymerase chain reaction, and tinctorial and immunohistochemical staining, using light and laser scanning confocal microscopy. Primary nasal epithelial cells were injured with reactive oxygen species and bacterial ligands to mimic epithelial damage in chronic rhinosinusitis, and the primary nasal epithelial cell pro-inflammatory cytokine release was measured. The cytokines were collected and transferred to cultured airway fibroblasts. The subsequent primary nasal epithelial cell induced pro-inflammatory fibroblast activation was measured and ways to block it were investigated.

\section{Results}

In this characterised ex vivo nasal epithelial-fibroblast model, we showed that injured nasal epithelial cells release the soluble pro-inflammatory cytokine interleukin (IL)-1a. This interleukin can chronically activate pro-inflammatory airway fibroblasts. We were also able to block this inflammation to a baseline level with either IL-1 $\alpha$ blocking antibodies or receptor antagonists.

\section{Conclusions}

We propose that sinonasal epithelial injury leads to the activation of inflammatory fibroblasts. These fibroblasts may ultimately drive a perpetual cycle of inflammation and fibrosis. This airway remodelling cycle is pathognomonic of chronic sinusitis. We were able to block the IL-1 $\alpha$ mediated nasal inflammation to a baseline level using agents known to be safe to humans. The findings may lead the way for a novel sinonasal anti-inflammatory treatment.
The management of $\mathbf{1 3}$ type III laryngeal clefts in children with open surgery: is elective tracheostomy needed? A change in practice

\author{
A Hay, R Hewitt, B Hartley \\ From Great Ormond Street Hospital for Children, London
}

\section{Introduction}

Laryngeal clefts are congenital midline abnormalities of the larynx. They are characterised by abnormal posterior sagittal communication between the larynx and the pharynx that can extend inferiorly down to the carina and oesophagus in severe cases. They are commonly staged into four grades of severity.

We describe the role of open surgical repair of type III clefts (and the associated advancement), and discuss its place in the pre-operative evaluation and management strategies adopted. These strategies include: repairing the cleft through a laryngofissure approach, without avascular grafts; assessment with video fluoroscopy swallowing tests; reducing the use of elective tracheostomy; and the aggressive treatment of gastro-oesophageal reflux prior to cleft repair.

\section{Method}

A retrospective search was carried out of the ENT surgery database at our institution in order to identify all surgery performed for laryngeal clefts between January 2002 and December 2012. All type III laryngeal clefts treated with open surgery and without tracheostomy were reviewed. The data collected included: demographic information, associated diagnoses or syndromes, surgical technique, use of tracheostomy and gastrostomy, findings of video fluoroscopy swallowing imaging, and outcomes including swallowing results, length of hospital stay and surgical complications.

\section{Results}

A total of 13 grade III laryngeal clefts treated with open surgery and without elective tracheostomy were identified. The operations were performed between November 2004 and December 2012. The mean patient age was 2.3 years (ranging from 18 months, to 4 years and 10 months) with 7 males and 6 females.

Twelve cases were associated with a known syndrome or severe congenital abnormality. Four patients had been diagnosed with Opitz G syndrome; five with vertebral anomalies, anal atresia, cardiac defects, tracheoesophageal fistula and/ or oesophageal atresia, renal anomalies and limb defects ('VACTERL') syndrome; two with congenital diaphragmatic hernias; and one with a tracheoesophageal fistula and a laryngeal cleft. One patient had severe gastro-oesophageal reflux. Elective gastrostomy was performed in nine patients; five of these patients underwent Nissen fundoplication prior to laryngeal cleft repair. No tracheostomy procedures were performed. Three patients required revision surgery.

With regard to treatment outcomes, nine patients were on an oral diet, three were still using a gastrostomy tube but their fluoroscopic swallow results had improved, and one continued to have an unsafe swallow. There were no major surgical complications.

\section{Discussion and conclusion}

The management of laryngeal clefts is evolving. We have shown that severe laryngeal clefts (type III) can be managed safely via open surgery to the airway, without elective tracheostomy. Pre-operative assessment and aggressive management of gastroesophageal reflux are seen as important pre-requisites for successful surgery. 
Aspirin desensitisation for Samter's triad: effect on health status

\section{R Kumar, D J Willatt \\ From the Salford Royal Foundation Trust}

\section{Introduction}

Aspirin desensitisation therapy is an under-utilised treatment modality for patients with Samter's triad. The recalcitrant nature of the disease limits the benefit of traditional treatments (which often fail to resolve symptoms), leads to early recurrence and multiple operations, and has a significant impact on quality of life. This study aimed to establish the change in health status (as measured by Glasgow Benefit Inventory) in patients with Samter's triad who have undergone aspirin desensitisation therapy for refractory nasal polyposis.

\section{Materials and methods}

All the Samter's triad patients who had undergone aspirin desensitisation therapy in our department were identified and contacted via telephone. These patients were asked if they would mind completing the Glasgow Benefit Inventory, either via a telephone interview or using a written format.

\section{Results}

Nine patients were identified, of which four were male and five were female. Mean patient age was 42 years. The mean follow-up interval was 16 months \pm 6 months. An average of $3.5 \pm 1.5$ surgical procedures had been undertaken. Sustained symptom relief was achieved in five patients. Two patients completed the treatment but gained no perceived benefit, and the remaining two patients were unable to tolerate the treatment.

The average Glasgow Benefit Inventory scores for those who achieved sustained benefit were: $36.5 \pm 21.5$ for general benefits, 0 for social benefits and $25 \pm 8$ for physical health benefits. Three patients achieved resolution of previously persistent anosmia. None of the patients required revision surgery. For those patients who did not improve, the score was 0 for all categories. No negative outcomes were recorded. No adverse events occurred and no long-term side effects have been reported so far.

\section{Conclusion}

The Glasgow Benefit Inventory outcomes for aspirin desensitisation therapy in Samter's triad patients are favourable and comparable to scores following middle-ear surgery for hearing. We therefore feel that aspirin desensitisation therapy should be offered to all suitable patients with refractory symptoms. Although some patients gained no benefit, no patients reported any negative outcomes. The cost saving benefit of aspirin versus surgery to both the patient and the health system should also be considered.

The incidence of pharyngocutaneous fistulae following laryngectomy: a case series and meta-analysis

\section{Sayles, L Harrison, N Beasley, D Grant \\ From the Queen's Medical Centre, Nottingham University Hospitals} NHS Trust

\section{Background and objectives}

Pharyngocutaneous fistula is a serious complication following laryngectomy, and is associated with significant morbidity and mortality. The incidence of pharyngocutaneous fistula is increased in salvage laryngectomy compared with primary laryngectomy. This study aimed to quantify the incidence of pharyngocutaneous fistula following major laryngeal surgery in a large cohort of patients, and to identify potential risk factors for the development of pharyngocutaneous fistula in an era of organ preservation treatment for laryngeal carcinoma.

\section{Methods}

A retrospective case note review was conducted of all patients who underwent laryngectomy during an 11-year period between 2000 and 2011. The primary outcome measure was the development of a pharyngocutaneous fistula. Secondary outcomes were: time until resolution, requirement for surgical closure and fistula-related mortality. Other variables recorded were: disease stage, diabetic status, known vascular disease, previous chemoradiotherapy or radiotherapy alone, pre-operative serum albumin levels, and pre- and post-operative haemoglobin levels.

Univariate statistical analyses were conducted using Fisher's exact test, chi-square test and student's $t$-test. A multivariate logistic model was fitted to the data. A metaanalysis was performed (using a random-effects model) on data from 25 previous studies in order to quantify the reported incidence of pharyngocutaneous fistula, stratified by the extent of surgical resection and initial treatment modality.

Results

Data were collected from 147 patients who underwent laryngeal resection in a tertiary referral setting. Of these patients, 86 had a standard total laryngectomy, 29 had a total laryngectomy and partial pharyngectomy, 11 underwent total laryngectomy and total pharyngectomy, 19 had a total pharyngo-laryngo-oesophagectomy with gastric transposition, and 2 underwent partial laryngectomy. Seventy-eight cases were primary surgical resections. Seventy-three cases were salvage resections following failed organ preservation treatment with either radiotherapy alone, or combined chemoradiotherapy.

The overall incidence of pharyngocutaneous fistula in the patient cohort was: standard total laryngectomy, 22 of 86 ( 25.6 per cent) ( 6 out of 40 of these patients (15 per cent) underwent primary total laryngectomy and 16 of 46 (34.7 per cent) underwent salvage total laryngectomy); total laryngectomy and total pharyngectomy, 3 of 11 (27.2 per cent); total laryngectomy and partial pharyngectomy, 11 of 29 (37.9 per cent); total pharyngo-laryngo-oesophagectomy with gastric transposition, 9 of 19 (47.3 per cent); and partial laryngectomy, 0 of 2 ( 0 per cent). Pharyngocutaneous fistula incidence was significantly increased in cases that involved salvage compared with primary surgery: 34.2 per cent and 24.4 per cent, respectively (chi-square $=3.85$, degrees of freedom $=1, p=0.04$ ). Pre-operative hypoalbuminaemia $(<35 \mathrm{~g} / 1)$ was the only variable significantly correlated with an increased incidence of pharyngocutaneous fistula on univariate analyses $(p<0.05)$. Multivariate analysis showed a complex interaction of variables.

The meta-analysis of data from 3470 patients in 25 studies showed that the overall incidence of pharyngocutaneous fistula was 31 per cent (95 per cent confidence interval (CI), 26-36 per cent) for salvage total laryngectomy with primary pharyngeal closure, and 14.9 per cent $(95$ per cent CI, 12.2-17.7 per cent) for primary total laryngectomy with primary pharyngeal closure.

\section{Conclusions}

These data confirm a significantly increased risk of pharyngocutaneous fistula in salvage surgery. There was a complex 
interaction of factors; pharyngocutaneous fistula incidence was related to co-morbid status, disease stage and extent of resection. A meta-analysis of data from a large number of patients undergoing laryngectomy for squamous cell carcinoma allowed an accurate prediction of the likely risk of pharyngocutaneous fistula formation.

\section{Airway stenosis in granulomatosis with polyangiitis (Wegener's granulomatosis) - assessment and management}

M Martinez Del Pero, D Jayne, A Chaudhry, P Sivasothy, P Jani

From Addenbrooke's Hospital, Cambridge University Hospitals

Objectives

To assess the demography, extent of disease and treatment outcome of airway stenosis in granulomatosis with polyangiitis (Wegener's granulomatosis).

\section{Methods}

All patients treated at a tertiary centre between 1997 and 2012 were included. Demographic data were recorded at baseline and at time of treatment. Details of each intervention were noted. Remission was defined as 12 months or more without intervention.

\section{Results}

Forty-four patients with granulomatosis with polyangiitis, and airway stenosis, were included. The median age at diagnosis was 37.6 years, and 72.7 per cent were female. The median duration of follow up was 62.5 months and the median duration of follow up following the last treatment was 27 months.

Subglottic stenosis was found in 36 patients and lower airway stenosis was found in 30 patients. The median number of lesions was 2 , and 11 patients had an isolated stenosis. There were 213 interventions recorded, including balloon dilatation, bougies and the use of a carbon dioxide laser. At least 1 episode of remission occurred in 97.4 per cent of patients ( 38 of 39 patients) after a median number of 2 procedures (interquartile range 1-6).

Fourteen adverse events were recorded ( 6.6 per cent). No tracheostomies were performed in the last five years.

\section{Conclusions}

The treatment of airway stenosis in granulomatosis with polyangiitis is complex; successful treatment requires careful medical management and repeated procedures. Hot and cold techniques, with or without adjuvant topical medication (including mitomycin $\mathrm{C}$ ), are safe. This report shows that a structured approach to these patients provides benefits in terms of achieving periods of remission. Based on this experience and the findings of previous reports, we propose an algorithm to manage this condition.
Injection laryngoplasty performed under local anaesthetic in the out-patient setting: comparative voice outcomes

J Powell, P Carding, J A Wilson

From the Freeman Hospital, Newcastle upon Tyne

\section{Objectives}

To examine voice outcomes in patients undergoing endoscopic vocal fold medialisation by transcutaneous injection under local anaesthetic (LA), and compare these with outcomes previously observed for general anaesthetic (GA) Bioplastique ${ }^{\mathrm{TM}}$ injection laryngoplasty and Isshiki thyroplasty.

\section{Study design}

Case-control study.

\section{Subjects and method}

Sixty-eight patients underwent LA injection laryngoplasty in the out-patient clinic over the two-year study period. The scores for a voice performance (patient-rated) questionnaire and perceptual voice analysis (observer-rated) scale (i.e. the grade, roughness, breathiness, asthenia and strain ('GRBAS') scale) were recorded before and after injection laryngoplasty. These were compared to previously published voice outcome data on GA Bioplastique laryngoplasty and thyroplasty procedures.

\section{Results}

Of the 68 patients, 57 had sufficient pre- and post-operative data to be included in the analysis. These included 32 men and 25 women, aged between 26 and 97 years. The remaining patients were excluded because: a procedure failed $(n=$ 3 ), they were too unwell to proceed $(n=1)$, they died before follow up was complete $(n=3)$ or their data were incomplete $(n=4)$.

Median voice performance questionnaire scores improved from 42 to $21(p<0.0001)$. Significant improvements were seen in all parameters of the grade, roughness, breathiness, asthenia and strain scale. These results were consistent with those of other studies following GA Bioplastique laryngoplasty and thyroplasty. Complication rates between the three procedures were also comparable.

\section{Conclusion}

Injection laryngoplasty performed in the out-patient clinic under LA led to improvements in subjective and perceptual voice outcomes; the results were similar to those obtained in the operating theatre under GA or sedation. This option is timely, cost-effective and obviates the need for general anaesthesia in patients, many of whom have co-morbidities and diminished life expectancy. 